\title{
Concha Bullosa in Paradoxical Middle Turbinate: A New Variation
}

\author{
${ }^{1}$ Mohammad W El-Anwar, ${ }^{2}$ Ahmed I Ali
}

\section{ABSTRACT}

Introduction: Concha bullosa is the most common anatomic variation of osteomeatal complex region that is generally seen in the middle turbinate (MT).

Materials and methods: A 25-year-old male presented with headache and nasal obstruction. Computed tomography (CT) scan documented right paradoxical MT. The right MT also showed aerated concha bullosa with narrow right osteomeatal area. Routine preoperative laboratory tests were within normal limits.

Results: This case of concha bullosa in paradoxically bent MT was reported, described, and could be safely managed endoscopically. Patient was symptom free up to date without any complication, recurrence, or other pathology.

Conclusion: Computed tomography may easily identify such uncommon anatomic variations of the osteomeatal region. This directs the surgeon attention to these variations as a cause of headache and osteomeatal area obstruction.

Keywords: Concha bullosa, Middle turbinate, Osteomeatal area.

How to cite this article: El-Anwar MW, Ali Al. Concha Bullosa in Paradoxical Middle Turbinate: A New Variation. Clin Rhinol An Int J 2016;9(3):141-142.

\section{Source of support: Nil}

Conflict of interest: None

\section{INTRODUCTION}

The middle turbinate (MT) projects downward over the ostia of the maxillary and ethmoid sinuses. The MT acts as a buffer to protect the sinuses from direct contact with pressurized nasal airflow. Most of the inhaled airflow passes between the inferior turbinate and MT.

Turbinate pneumatization (concha bullosa) refers to the presences of an air cell(s) inside the turbinate. Concha bullosa of the MT is a common variant that varies from

\footnotetext{
${ }^{1}$ Assistant Professor, ${ }^{2}$ Consultant

${ }^{1}$ Department of Otorhinolaryngology, Zagazig University Zagazig, Egypt

${ }^{2}$ Department of Otorhinolaryngology - Head and Neck Surgery Faculty of Medicine, Zagazig University, Zagazig, Egypt

Corresponding Author: Mohammad W El-Anwar, Assistant Professor, Department of Otorhinolaryngology, Zagazig University, Zagazig, Egypt, Phone: +0020552307830, e-mail: mwenteg@yahoo.com
}

14 to $53 \%,{ }^{2}$ and it represents the most common anatomic variation of osteomeatal complex region. ${ }^{3}$

A paradoxical MT refers to an inferomedially curved MT edge with the concave surface facing the nasal septum. ${ }^{2}$ It usually occurs bilaterally. This variant alone can cause significant narrowing of the middle meatus and impedes the normal drainage of paranasal sinuses due to osteomeatal complex and nasal obstruction., ${ }^{1,4}$

\section{CASE REPORT}

A 25-year-old male presented with headache and nasal obstruction for over 2 years with no epistaxis, sneezing, or smell affection. He had no history of facial trauma, nasal surgery, or significant medical history. Other otolaryngological and general examinations were normal.

Computed tomography (CT) scan showed paradoxical MT on both sides. The right MT also showed aerated concha bullosa with narrowed right osteomeatal area and maxillary ostium (Fig. 1). Routine preoperative laboratory tests were within normal limits.

After an informed consent form was signed by the patient, under general anesthesia, the paradoxically bent part of the MT was removed endoscopically, and then the lateral lamella of the concha bullosa was meticulously
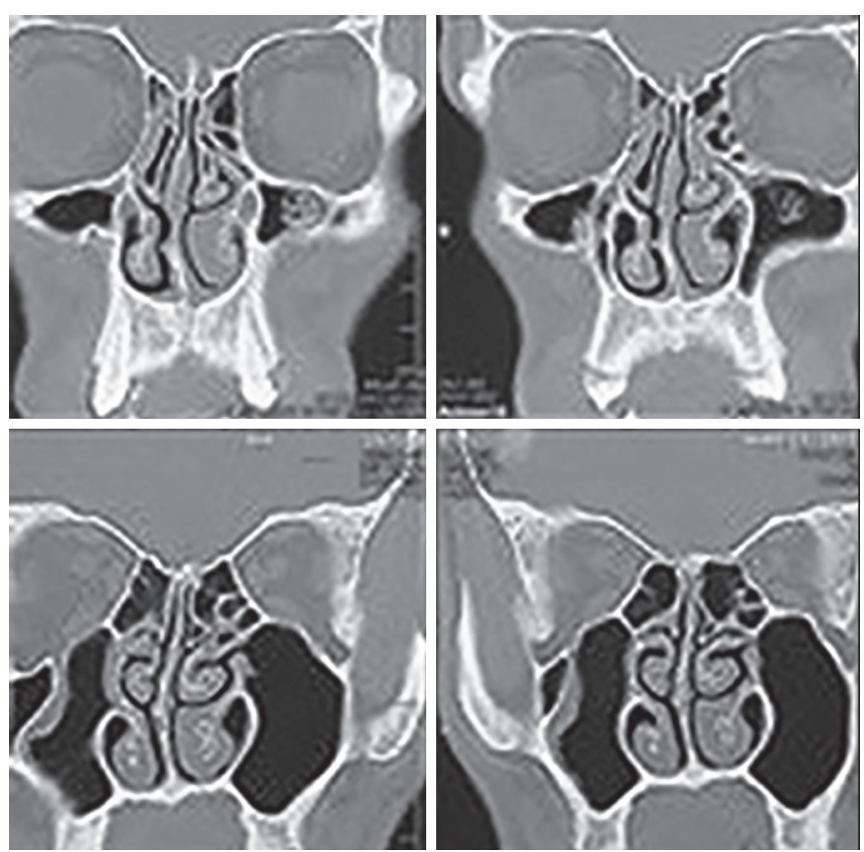

Fig. 1: Computed tomography showing bilateral paradoxically bent MT with right concha bullosa 

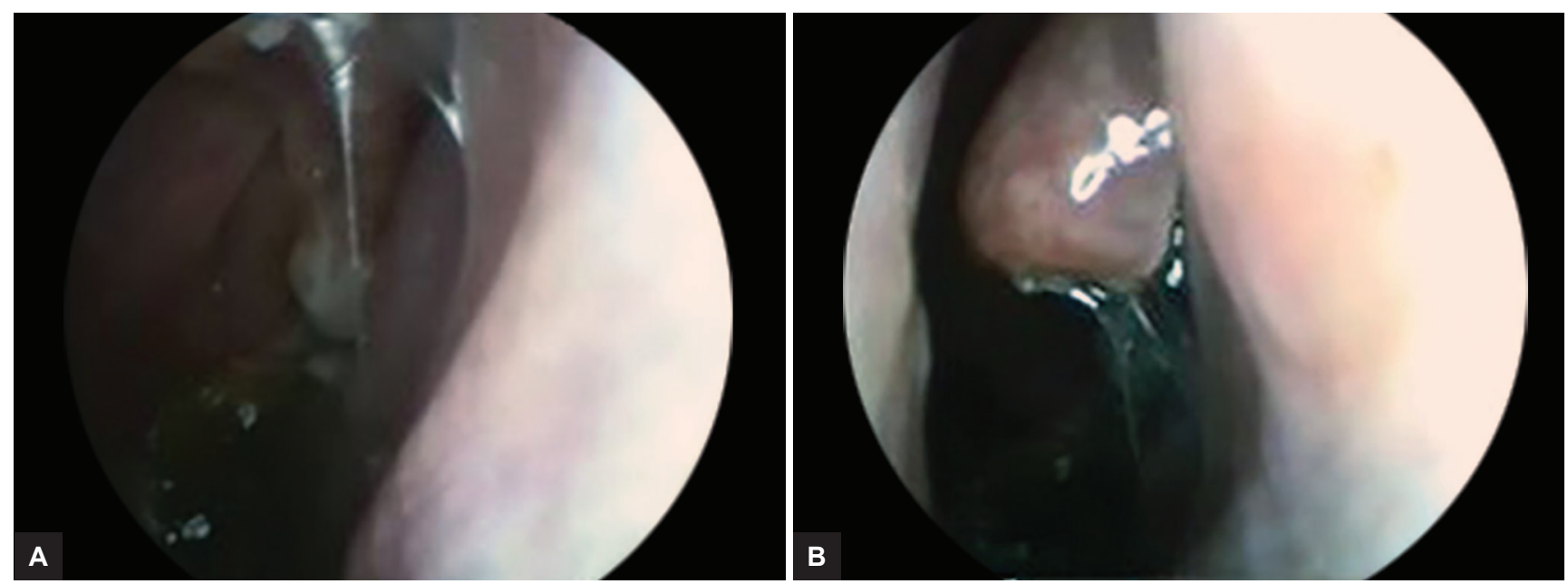

Figs 2A and B: One-month postoperative endoscopic view

removed. The stability of the MT was preserved at the end of surgery, and the osteomeatal area became patent with clearly seen bulla ethmoidalis and uncinate process. The postoperative course was uneventful, and the patient was discharged on the same day of surgery without any complaint.

The patient was satisfied and symptom free up to date without complication, recurrence, or other pathologies (Figs 2A and B).

\section{DISCUSSION}

Nasal causes of headaches include paradoxically bent MT and pneumatized turbinates. ${ }^{5}$ To the best of our knowledge, the concha bullosa was not previously described and treated in paradoxically bent MT.

The advancement of imaging techniques, such as CT has provided us detailed information of patients' anatomy. This helps the surgeons to be aware of the anatomic variations in the osteomeatal region. ${ }^{6}$

We reported a case with bilateral paradoxically bent MTs with right concha bullosa detected by CT. Endoscopic surgery was performed dealing with both variants with the aim to preserve stability and skull base attachment of the MT. So we carefully removed the paradoxical part of the MT first. Then surgical resection of concha bullosa entails careful preservation of medial lamella (which attaches to the skull base) and resection of only lateral half of turbinate as previously recommended by Braun and Stammberger for concha bullosa. ${ }^{6}$

Concha is common in a variant MT, where it is important to operate with precautions to avoid MT instability and skull base affection. But in the current case with also paradoxical MT, it is more difficult to achieve these goals. Moreover, the presence of both variants in the same MT could lead to misdetection of one of them.

Besides, it is more difficult to perform surgery to reserve the patency of the osteomeatal area while preserving the MT stability and skull base attachment.

The current study highlights the importance of diagnosing both variations in the MT and treating both of them to avoid persistent obstruction of the drainage area and patients' complaint.

To the best of our knowledge, this is the first study to report and describe a case of a paradoxical MT and treat it successfully endoscopically. Therefore, this combination should be considered during the CT.

\section{CONCLUSION}

A case of concha bullosa in paradoxically bent MT was reported, described, and could be safely managed endoscopically. Computed tomography could easily identify such anatomic variations of the osteomeatal region. This study directs surgeons' attention to variations as a cause of headache and osteomeatal obstruction.

\section{REFERENCES}

1. Pittore B, Al Safi W, Jarvis SJ. Concha bullosa of the inferior turbinate: an unusual cause of nasal obstruction. Acta Otorhinolaryngol Ital $2011 \mathrm{Feb} ; 31(1): 47-49$.

2. Ural A, Uslu SS, Ileri F, Atilla MH, Ozbilen S, Köybaşiŏglu A. Giant concha bullosa. Kulak Burun Bŏgaz ve Baş Boyun Cerrahisi Dergisi 2002;10:89-92.

3. Badia L, Lund VJ, Wei W, Ho WK. Ethnic variation in sinonasal anatomy on CT scanning. Rhinology 2005 Sep;43(3):210-214.

4. Neskey D, Eloy JA, Casiano RR. Nasal, septal, and turbinate anatomy and embryology. Otolaryngol Clin North Am 2009 Apr;42(2):193-205.

5. Braun $H$, Stammberger $H$. Pneumatization of turbinates. Laryngoscope 2003 Apr;113(4):668-672.

6. San T, ErdoLan B, TaGel B. Triple-divided concha bullosa: a new anatomic variation. Case Rep Otolaryngol 2013;2013:342615. 